\title{
Swapped (OTIS) Networks Built of Connected Basis Networks Are Maximally Fault Tolerant
}

\author{
Weidong Chen, Wenjun Xiao, and Behrooz Parhami, Fellow, IEEE
}

\begin{abstract}
An optical transpose interconnection system (OTIS) network with $n^{2}$ nodes is a two-level swapped architecture built of $n$ copies of an $n$-node basis network that constitute its clusters. A simple rule for intercluster connectivity (node $j$ in cluster $i$ connected to node $i$ in cluster $j$, for all $i \neq j$ ) leads to regularity, modularity, packageability, fault tolerance, and algorithmic efficiency of the resulting networks. We prove that an OTIS (swapped) network with a connected basis network possesses maximal fault tolerance, regardless of whether its basis network is maximally fault tolerant. We also show how the corresponding maximal number of node-disjoint paths between two nodes of a swapped network can be algorithmically constructed in a manner that is independent of the existence and construction of node-disjoint paths within its basis network. Our results complement previous studies that show swapped networks inheriting some desirable properties (e.g., Hamiltonicity) from their basis networks. Here, we show that the swapped connectivity actually introduces a desirable property that may not exist in the basis network. Furthermore, our general result about maximal connectivity and the corresponding node-disjoint path construction for swapped networks replace a number of proofs and constructions in the literature for specific basis networks and obviate the need for proving maximal fault tolerance and dealing with node-disjoint path constructions for many other basis networks of potential practical interest. Additionally, we use our parallel path constructions to establish that the fault diameter and wide diameter of an OTIS network is no more than 4 units greater than its diameter.
\end{abstract}

Index Terms-Connectivity, fault diameter, interconnection network, maximal fault tolerance, node-disjoint paths, OTIS network, parallel paths, robustness, swapped network, topological properties, wide diameter.

\section{INTRODUCTION}

$\mathrm{O}$ PTICAL transpose interconnection system (OTIS) networks were originally devised to provide efficient connectivity for new optoelectronic computer architectures that benefit from both optical and electronic technologies [6]. In OTIS networks, processors are arranged into clusters. Electronic interconnects are used between processors within the same cluster, while optical links are used for intercluster communication. It has been shown [5] that bandwidth and power consumption are optimized when the number of processors in a cluster equals the number of clusters. Thus, we limit our study to such $n^{2}$-node OTIS networks with $n$-node clusters [2], [10]. The OTIS architecture has received considerable attention in recent years and has a special place among real-world architectures for parallel and distributed systems [2], [3]. A number of algorithms have been developed for routing, selection/ sorting [4], [8], [11], [12], [15], certain numerical computations [7], Fourier transform [1], matrix multiplication [14], and image processing [13].

Studying properties of general composite interconnection schemes, such as OTIS (swapped) networks, is important in that it allows the derivation of results pertaining to a wide

- W. Chen and W. Xiao are with the Department of Computer Science, South China University of Technology, Guangzhou 510641, P.R. China. W. Chen is also with the Department of Computer Science, South China Normal University, Guangzhou 510631, P.R. China.

- B. Parhami is with the Department of Electrical and Computer Engineering, University of California, Santa Barbara, Santa Barbara, CA 93106-9560. E-mail: parhami@ece.ucsb.edu.

Manuscript received 5 Mar. 2008; revised 19 May 2008; accepted 21 May 2008; published online 28 May 2008.

Recommended for acceptance by X. Zhang.

For information on obtaining reprints of this article, please send e-mail to: tpds@computer.org, and reference IEEECS Log Number TPDS-2007-03-0068. Digital Object Identifier no. 10.1109/TPDS.2008.98. array of network architectures. Several general properties of OTIS networks have been considered [2], [10]. More recently, Parhami has established the Hamiltonicity of OTIS (swapped) networks built of Hamiltonian basis networks [9]. Fault tolerance of interconnection networks is among the properties of considerable interest in parallel and distributed computation. An important aspect of fault tolerance is the number of node-disjoint (or parallel) paths between nodes of the networks. Such parallel paths are useful in speeding up the transfer of large amounts of data between nodes and for providing alternate routes in cases of node or link failures. Hence, a network with many parallel paths is robust.

In this paper, we contribute further results in this direction by studying a general fault tolerance property of OTIS networks. We prove that an OTIS network is maximally fault tolerant if its basis network is connected, and propose a corresponding method for constructing parallel paths between its nodes. Because the use of disconnected basis networks is not practically viable, our result essentially shows that any OTIS network of practical interest is maximally fault tolerant. This is stronger than the corresponding result of [2], stating that an OTIS network is maximally fault tolerant if its basis network possesses maximal fault tolerance.

One contribution of our work is that our proof of the maximal fault tolerance property (the corresponding parallel path construction, respectively) leads to maximal fault tolerance results (parallel path construction) for a wide array of interconnection networks. For example, the recent result of Day [3] on the maximal fault tolerance of optical transpose $k$-ary $n$-cube networks follows as a special case of our result. Another contribution of our work is showing that an OTIS network enjoys some new desirable properties, such as maximal fault tolerance, independent of the fault 
tolerance of its basis network. This is fundamentally different from those results in the literature [2], [3], [9], [10] demonstrating that OTIS networks can inherit some desirable properties, such as short diameter and Hamiltonicity, from their basis networks.

\section{Basic Definitions and Related Work}

Let $G$ be a simple undirected graph (graph, for short) with the vertex set $V_{G}$ and the edge set $E_{G}$. For $v \in V_{G}$, we denote by $\operatorname{deg}_{G}(v)$ the degree of $v$ in $G$, by $\mathrm{N}_{G}(v)=\{u \in V \mid(v, u) \in$ $\left.E_{G}\right\}$ the open neighborhood of $v$, and by $\mathrm{N}_{G}[v]=$ $\mathrm{N}_{G}(v) \cup\{v\}$ its closed neighborhood. The maximum degree among the vertices of $G$ is denoted by $\Delta(G)$ and the minimum degree by $\delta(G)$. The distance between two nodes $u$ and $v$, denoted as $d_{G}(u, v)$, is the length of a shortest path between $u$ and $v$. The diameter $D(G)$ of $G$ is the maximal distance between any two nodes in $G$. Other notation and terminology used in this paper follow those in [16]. In the remainder of this paper, we use the terms graph and network interchangeably.

Definition 1 (OTIS (Swapped) network [6], [17], [18]). The OTIS (swapped) network OTIS- $\Omega$, derived from the graph $\Omega$, is a graph with vertex set $V_{\text {OTIS- } \Omega}=\left\{\langle g, p\rangle \mid g, p \in V_{\Omega}\right\}$ and edge set $E_{\mathrm{OTIS}-\Omega}=\left\{\left(\left\langle g, p_{1}\right\rangle,\left\langle g, p_{2}\right\rangle\right) \mid g \in V_{\Omega},\left(p_{1}, p_{2}\right) \in\right.$ $\left.E_{\Omega}\right\} \cup\left\{(\langle g, p\rangle,\langle p, g\rangle) \mid g, p \in V_{\Omega}\right.$ and $\left.g \neq p\right\}$.

In OTIS- $\Omega$, the graph $\Omega$ is called the basis (factor) graph or network. If the basis network $\Omega$ has $n$ nodes, then OTIS- $\Omega$ is composed of $n$ node-disjoint subnetworks called clusters, each of which is isomorphic to $\Omega$. The node label $\langle g, p\rangle$ in OTIS- $\Omega$ identifies the node indexed $p$ in cluster $g$. We refer to $g$ as the cluster address of node $\langle g, p\rangle$ and $p$ as its processor address.

In such an OTIS network, an intercluster or swap (optical) link connects processor $p$ of cluster $g$ to processor $g$ of cluster $p$, for all $p \neq g$. No intercluster link is incident to processor $g$ of cluster $g$. All the intracluster (electronic) links in a cluster form an interconnection topology corresponding to the basis graph. Fig. 1 depicts example OTIS networks with the 4-node cycle $C_{4}$ and the 6-node complete graph $K_{6}$ as the basis graphs.

The following topological parameters of OTIS- $\Omega$, needed later in this paper, are easily derived from Definition 1. These OTIS- $\Omega$ parameters have been formulated as functions of the corresponding metrics of $\Omega$ :

- $\quad \operatorname{deg}_{\mathrm{OTIS}-\Omega}(\langle g, g\rangle)=\operatorname{deg}_{\Omega}(g)$.

- $\quad$ For $g \neq p$, $\operatorname{deg}_{\text {OTIS- } \Omega}(\langle g, p\rangle)=\operatorname{deg}_{\Omega}(p)+1$.

- $\Delta($ OTIS $-\Omega)=\Delta(\Omega)+1$.

- $\delta($ OTIS- $\Omega)=\delta(\Omega)$.

- $\quad D($ OTIS $-\Omega)=2 D(\Omega)+1$.

There are two main measures for good connectivity in networks: invulnerability to deletions and multiplicity of alternate paths.

Definition 2 (Connectivity [16]). The connectivity $k(G)$ of a graph $G$ is the minimum size of a node set $S$ such that $G-S$ is either disconnected or has a single node. A graph $G$ is $c$-connected if its connectivity is at least $c$.

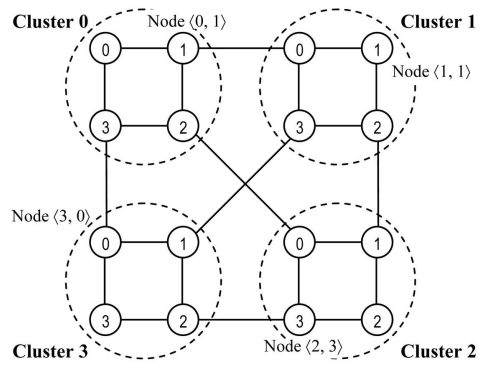

(a)

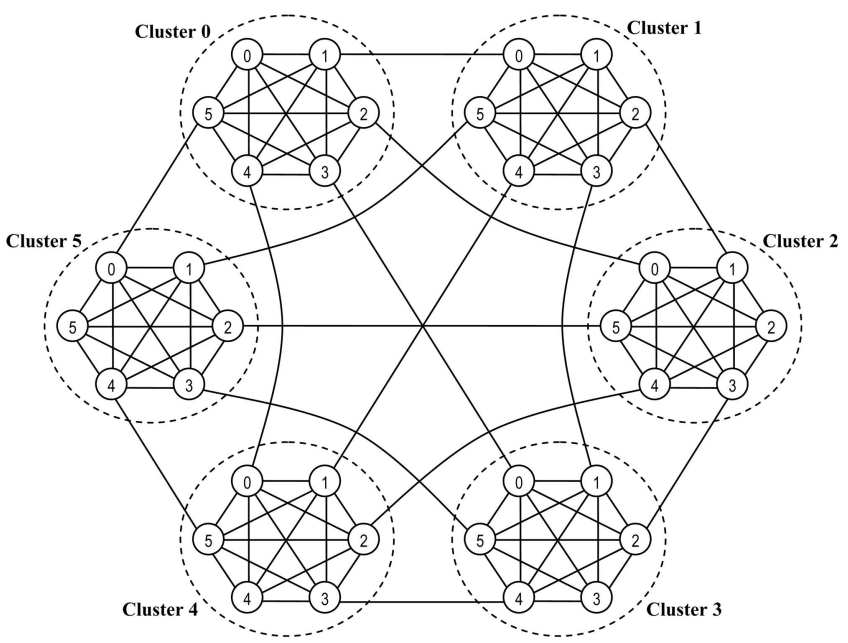

(b)

Fig. 1. Two example OTIS networks, one built of the 4-node cycle $C_{4}$ as the basis network and the other based on the 6-node complete graph $K_{6}$. A few node labels are shown on the first network as examples. (a) OTIS- $C_{4}$. (b) OTIS- $K_{6}$.

The connectivity $k(G)$ of $G$ always satisfies the inequality $k(G) \leq \delta(G)$ [16].

Definition 3 (Fault tolerance [16]). In a graph $G$, for $u$, $v \in V_{G}$, a path from $u$ to $v$ is called $a(u, v)$-path for short. Two $(u, v)$-paths are node-disjoint if they have no common internal node. A measure of network fault tolerance is the existence of alternative paths between nodes: the more node-disjoint paths, the better.

One of Menger's fundamental theorems in graph theory (see [16, p. 169], for example) states that there exists a close relationship between the notions of connectivity and fault tolerance of a graph.

Theorem 1 (Menger's theorem). The connectivity of a graph $G$ equals the maximum $k$ such that $\lambda(x, y) \geq k$ for all $x, y \in V_{G}$, where $\lambda(x, y)$ is the maximum number of (pairwise) nodedisjoint $(x, y)$-paths.

Therefore, the fault tolerance of a network can be measured by its connectivity, and a network is maximally fault tolerant if its connectivity equals its minimum node degree.

The following results about the fault tolerance of an OTIS network have been proven by Day and Al-Ayyoub [2].

Theorem 2 (Connectivity of OTIS- $\Omega$ ). Let the graph $\Omega$ be connected: 


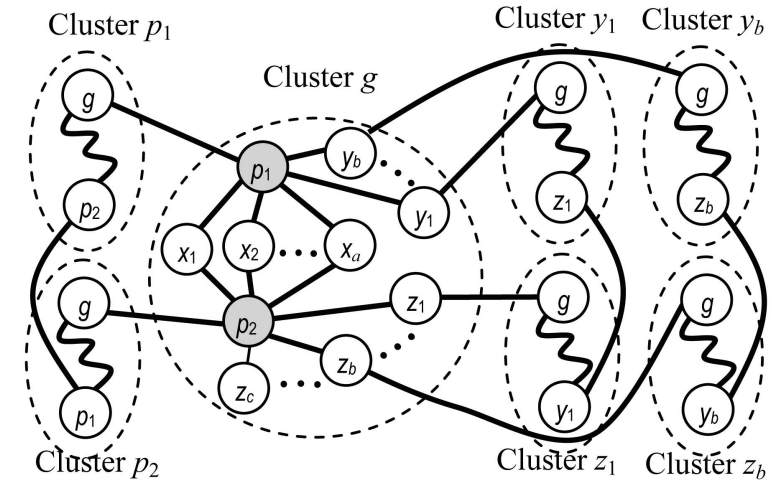

Fig. 2. Constructing $d+1$ node-disjoint paths, shown as heavy lines, in OTIS- $\Omega$ between $\left\langle g, p_{1}\right\rangle$ and $\left\langle g, p_{2}\right\rangle$ for $p_{1} \neq p_{2}$, $\mathrm{N}_{\Omega}\left[p_{1}\right] \cap \mathrm{N}_{\Omega}\left[p_{2}\right] \neq \emptyset$, and $g \notin \mathrm{N}_{\Omega}\left[p_{1}\right] \cup \mathrm{N}_{\Omega}\left[p_{2}\right]$, where $s_{1}=s_{2}=x_{1}$, $X=\left\{x_{2}, \ldots, x_{a}\right\}, \quad Y=\left\{p_{1}, y_{1}, \ldots, y_{b}\right\}, \quad Z=\left\{p_{2}, z_{1}, \ldots, z_{b}, \ldots, z_{c}\right\}$, and $M=\left\{\left(p_{1}, p_{2}\right),\left(y_{1}, z_{1}\right), \ldots, \quad\left(y_{b}, z_{b}\right)\right\}$.

1. If $\left\langle g_{1}, p\right\rangle$ and $\left\langle g_{2}, p\right\rangle$ are two nodes in OTIS- $\Omega$ such that $g_{1} \neq g_{2}$ and $\operatorname{deg}_{\Omega}(p)=d$, then there are $d$ nodedisjoint $\left(\left\langle g_{1}, p\right\rangle,\left\langle g_{2}, p\right\rangle\right)$-paths in OTIS- $\Omega$.

2. If $\left\langle g_{1}, p_{1}\right\rangle$ and $\left\langle g_{2}, p_{2}\right\rangle$, with $p_{1} \neq p_{2}$, are two nodes in OTIS- $\Omega$, such that there are $d$ node-disjoint $\left(p_{1}, p_{2}\right)$-paths in $\Omega$, then there are $d$ node-disjoint $\left(\left\langle g_{1}, p_{1}\right\rangle,\left\langle g_{2}, p_{2}\right\rangle\right)$-paths in OTIS- $\Omega$.

The following corollary readily follows from Theorem 2.

Corollary 1. OTIS- $\Omega$ is maximally fault tolerant if the basis network $\Omega$ is maximally fault tolerant.

\section{Proof of Maximal Fault Tolerance}

In this section, we provide an algorithmic construction of node-disjoint paths between nodes in OTIS- $\Omega$, with $\Omega$ being connected, which is straightforward and independent of node-disjoint path constructions between nodes in $\Omega$. By showing that OTIS- $\Omega$ contains at least $\delta$ (OTIS- $\Omega$ ) nodedisjoint paths between two nodes, we prove that OTIS- $\Omega$ enjoys the maximal fault tolerance property, regardless of whether its basis network $\Omega$ is maximally fault tolerant. In the following, we establish this result by proving two main subcases in Lemmas 1 and 2 .

We begin by introducing several additional notational tools needed throughout this section. Let $Y$ and $Z$ be two disjoint subsets of $V_{\Omega}$. A maximal match $M$ from $Y$ to $Z$ is a subset of $Y \times Z$, with $|M|=\min \{|Y|,|Z|\}$, such that for any two distinct $(y, z),\left(y^{\prime}, z^{\prime}\right) \in M$, we have $y \neq y^{\prime}$ and $z \neq z^{\prime}$. We denote by $p \rightarrow q$ a link from $p$ to $q$ in $\Omega$, by $\langle g, p\rangle \rightarrow\langle p, g\rangle$ a swap link from $\langle g, p\rangle$ to $\langle p, g\rangle$ in OTIS- $\Omega$, where $g \neq p$, and by $\left\langle g, p_{1} \rightarrow p_{2} \rightarrow \cdots \rightarrow p_{h}\right\rangle$ or $\left\langle g, p a t h_{\Omega}\left(p_{1}, p_{h}\right)\right\rangle$ a path $\left\langle g, p_{1}\right\rangle,\left\langle g, p_{2}\right\rangle, \ldots,\left\langle g, p_{h}\right\rangle$ in OTIS- $\Omega$ that is completely contained in cluster $g$, with $p_{1}, p_{2}, \ldots, p_{h}$ forming a shortest path from $p_{1}$ to $p_{h}$ in $\Omega$. In addition, to achieve consistency and brevity, null links such as $p \rightarrow p$ and $\langle g, p\rangle \rightarrow\langle g, p\rangle$ are allowed in representing paths.

Lemma 1. Let $\Omega$ be a connected graph. If $\left\langle g, p_{1}\right\rangle$ and $\left\langle g, p_{2}\right\rangle$ are nodes in OTIS- $\Omega$ such that $p_{1} \neq p_{2}$, then there are $d$ or $d+1$ node-disjoint $\left(\left\langle g, p_{1}\right\rangle,\left\langle g, p_{2}\right\rangle\right)$-paths in OTIS- $\Omega$, where $d=\min \left\{\operatorname{deg}_{\Omega}\left(p_{1}\right), \operatorname{deg}_{\Omega}\left(p_{2}\right)\right\}$.

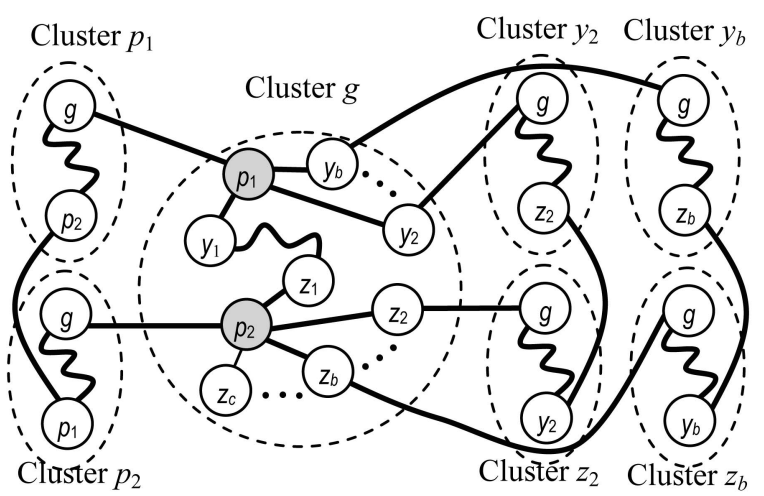

Fig. 3. Constructing $d+1$ node-disjoint paths, shown as heavy lines, in OTIS- $\Omega$ between $\left\langle g, p_{1}\right\rangle$ and $\left\langle g, p_{2}\right\rangle$ for $p_{1} \neq p_{2}, \mathrm{~N}_{\Omega}\left(p_{1}\right) \cap \mathrm{N}_{\Omega}\left(p_{2}\right)=\emptyset$, and $g \notin \mathrm{N}_{\Omega}\left[p_{1}\right] \cup \mathrm{N}_{\Omega}\left[p_{2}\right]$, where $s_{1}=y_{1}, s_{2}=z_{1}, X=\emptyset, Y=\left\{p_{1}, y_{2}, \ldots, y_{b}\right\}$, $Z=\left\{p_{2}, z_{2}, \ldots, z_{b}, \ldots, z_{c}\right\}$, and $M=\left\{\left(p_{1}, p_{2}\right),\left(y_{2}, z_{2}\right), \ldots,\left(y_{b}, z_{b}\right)\right\}$.

Proof. Without loss of generality, we assume $\operatorname{deg}_{\Omega}\left(p_{1}\right) \leq$ $\operatorname{deg}_{\Omega}\left(p_{2}\right)$. Then, we have $\left|N_{\Omega}\left(p_{1}\right)\right|=d,\left|N_{\Omega}\left[p_{1}\right]\right|=d+1$, $\left|N_{\Omega}\left(p_{2}\right)\right| \geq d$ and $\left|N_{\Omega}\left[p_{2}\right]\right| \geq d+1$. We next construct $d$ or $d+1$ paths between $\left\langle g, p_{1}\right\rangle$ and $\left\langle g, p_{2}\right\rangle$ in OTIS- $\Omega$, and, at the end, show these paths to be pairwise node-disjoint.

First, we construct the following shortest path from $\left\langle g, p_{1}\right\rangle$ to $\left\langle g, p_{2}\right\rangle$ based on a shortest path from $p_{1}$ to $p_{2}$ in $\Omega$ :

$$
\left\langle g, \operatorname{path}_{\Omega}\left(p_{1}, p_{2}\right)\right\rangle
$$

where $\operatorname{path}_{\Omega}\left(p_{1}, p_{2}\right)=p_{1} \rightarrow \operatorname{path}_{\Omega}\left(s_{1}, s_{2}\right) \rightarrow p_{2}$ for some $s_{1} \in \mathrm{N}_{\Omega}\left(p_{1}\right)$ and some $s_{2} \in \mathrm{N}_{\Omega}\left(p_{2}\right)$. In the special case of $p_{2} \in \mathrm{N}_{\Omega}\left(p_{1}\right)$, we have $\operatorname{path}_{\Omega}\left(p_{1}, p_{2}\right)=p_{1} \rightarrow p_{2}, s_{1}=p_{2}$, and $s_{2}=p_{1}$. Define $X=\mathrm{N}_{\Omega}\left[p_{1}\right] \cap \mathrm{N}_{\Omega}\left[p_{2}\right]-\left\{s_{1}, s_{2}\right\}$, $Y=\mathrm{N}_{\Omega}\left[p_{1}\right]-X-\left\{s_{1}, g\right\}$, and $Z=\mathrm{N}_{\Omega}\left[p_{2}\right]-X-\left\{s_{2}, g\right\}$. It is readily verified that $X, Y$, and $Z$ are pairwise disjoint sets, and that we have $X \subset \mathrm{N}_{\Omega}\left[p_{1}\right]$ and $X \subset \mathrm{N}_{\Omega}\left[p_{2}\right]$. Let $M$ be a maximal match from $Y$ to $Z$. Then, we construct $|X|+|M|$ paths from $\left\langle g, p_{1}\right\rangle$ to $\left\langle g, p_{2}\right\rangle$ as follows: For every $x \in X$, we have the paths

$$
\left\langle g, p_{1} \rightarrow x \rightarrow p_{2}\right\rangle
$$

and for every $(y, z) \in M$, we build the paths

$$
\begin{aligned}
\left\langle g, p_{1} \rightarrow y\right\rangle & \rightarrow\left\langle y, \operatorname{path}_{\Omega}(g, z)\right\rangle \rightarrow\left\langle z, \operatorname{path}_{\Omega}(y, g)\right\rangle \\
& \rightarrow\left\langle g, z \rightarrow p_{2}\right\rangle .
\end{aligned}
$$

The constructions in two typical cases are depicted in Fig. 2 (when $\mathrm{N}_{\Omega}\left[p_{1}\right] \cap \mathrm{N}_{\Omega}\left[p_{2}\right] \neq \emptyset$ ) and Fig. 3 (when $\left.\mathrm{N}_{\Omega}\left[p_{1}\right] \cap \mathrm{N}_{\Omega}\left[p_{2}\right]=\emptyset\right)$.

By the definitions of $X$ and $M$, the number of paths given in (1)-(3) above is $1+|X|+|M|$. Considering $\left|\mathrm{N}_{\Omega}\left[p_{1}\right]\right|=d+1, X \subset \mathrm{N}_{\Omega}\left[p_{1}\right], s_{1} \in \mathrm{N}_{\Omega}\left[p_{1}\right]$, and $s_{1} \notin X$, we have $|Y|=d-|X|-1$ if $g \in \mathrm{N}_{\Omega}\left[p_{1}\right]-X-\left\{s_{1}\right\}$; otherwise, $|Y|=d-|X|$. Similarly, because $\left|\mathrm{N}_{\Omega}\left[p_{2}\right]\right| \geq d+1$, we have $|Z| \geq d-|X|-1$ if $g \in \mathrm{N}_{\Omega}\left[p_{2}\right]-X-\left\{s_{2}\right\}$; otherwise, $|Z| \geq d-|X|$. Hence, $|M|=d-|X|-1$ or $d-|X|$ due to $|M|=\min \{|Y|,|Z|\}$, and the number $1+|X|+$ $|M|$ of paths is $d$ or $d+1$, as claimed. In particular, if $g \notin \mathrm{N}_{\Omega}\left[p_{1}\right] \cup \mathrm{N}_{\Omega}\left[p_{2}\right]$, then $|M|=d-|X|$, and the number of paths reaches its maximum of $d+1$.

To complete the proof, we must show the paths defined in (1)-(3) to be pairwise node-disjoint. Because $X, Y$, and $Z$ are pairwise disjoint sets, no cluster, other than cluster $g$, is 


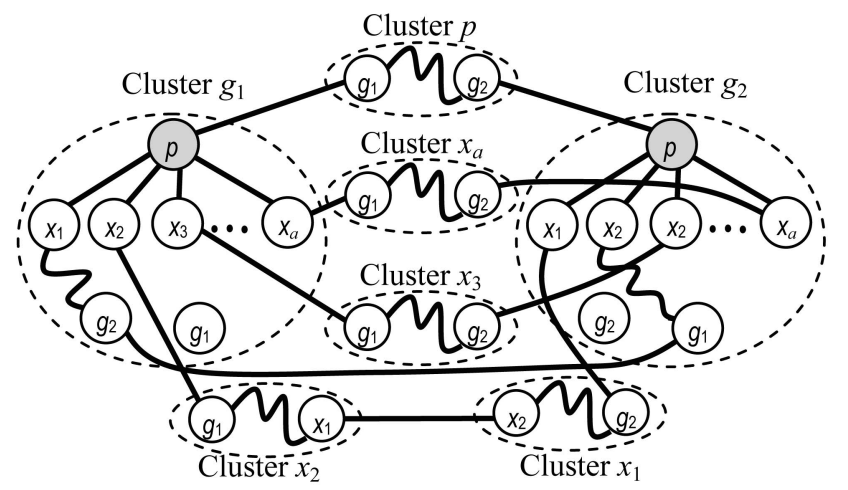

Fig. 4. Constructing $d+1$ node-disjoint paths, shown as heavy lines, in OTIS- $\Omega$ between $\left\langle g_{1}, p\right\rangle$ and $\left\langle g_{2}, p\right\rangle$ for $g_{1} \neq g_{2}$, and $g_{1}, g_{2} \notin \mathrm{N}_{\Omega}[p]$, where $s_{1}=x_{1}, s_{2}=x_{2}, X=\left\{p, x_{3}, \ldots, x_{a}\right\}, Y=\left\{x_{2}\right\}, Z=\left\{x_{1}\right\}$, and $M=\left\{\left(x_{2}, x_{1}\right)\right\}$.

visited by more than one of these paths. So, if any pair of paths were not node-disjoint, they would only intersect in cluster $g$. However, the latter is impossible, given that all segments of these paths contained in cluster $g$ are pairwise node-disjoint by construction.

Lemma 2. Let $\Omega$ be a connected graph. If $\left\langle g_{1}, p_{1}\right\rangle$ and $\left\langle g_{2}, p_{2}\right\rangle$ are nodes in OTIS- $\Omega$ such that $g_{1} \neq g_{2}$, then there are $d$ or $d+1$ node-disjoint $\left(\left\langle g_{1}, p_{1}\right\rangle,\left\langle g_{2}, p_{2}\right\rangle\right)$-paths in OTIS- $\Omega$, where $d=\min \left\{\operatorname{deg}_{\Omega}\left(p_{1}\right), \operatorname{deg}_{\Omega}\left(p_{2}\right)\right\}$.

Proof. Assume, without loss of generality, that $\operatorname{deg}_{\Omega}\left(p_{1}\right) \leq$ $\operatorname{deg}_{\Omega}\left(p_{2}\right)$. We then have $\left|N_{\Omega}\left(p_{1}\right)\right|=d,\left|N_{\Omega}\left[p_{1}\right]\right|=d+1$, $\left|N_{\Omega}\left(p_{2}\right)\right| \geq d$, and $\left|N_{\Omega}\left[p_{2}\right]\right| \geq d+1$. We next construct $d$ or $d+1$ paths between $\left\langle g_{1}, p_{1}\right\rangle$ and $\left\langle g_{2}, p_{2}\right\rangle$ in OTIS- $\Omega$, and, at the end, show these paths to be pairwise node-disjoint.

First, we construct the following path from $\left\langle g_{1}, p_{1}\right\rangle$ to $\left\langle g_{2}, p_{2}\right\rangle$ based a shortest path from $p_{1}$ to $g_{2}$ and a shortest path from $g_{1}$ to $p_{2}$ in $\Omega$ :

$$
\left\langle g_{1}, \operatorname{path}_{\Omega}\left(p_{1}, g_{2}\right)\right\rangle \rightarrow\left\langle g_{2}, \operatorname{path}_{\Omega}\left(g_{1}, p_{2}\right)\right\rangle,
$$

where $\operatorname{path}_{\Omega}\left(p_{1}, g_{2}\right)=p_{1} \rightarrow \operatorname{path}_{\Omega}\left(s_{1}, g_{2}\right)$ for some $s_{1} \in$ $\mathrm{N}_{\Omega}\left[p_{1}\right]$ and path $_{\Omega}\left(g_{1}, p_{2}\right)=$ path $_{\Omega}\left(g_{1}, s_{2}\right) \rightarrow p_{2}$ for some $s_{2} \in \mathrm{N}_{\Omega}\left[p_{2}\right]$. We should point out that in the special case of $p_{1}=g_{2}, \operatorname{path}_{\Omega}\left(p_{1}, g_{2}\right)=p_{1} \rightarrow p_{1}$ is null, and we have $s_{1}=p_{1}$. Similarly, in the special case of $g_{1}=p_{2}$, path $_{\Omega}\left(g_{1}, p_{2}\right)=p_{2} \rightarrow p_{2}$ is null, and we have $s_{2}=p_{2}$. Define $X=\mathrm{N}_{\Omega}\left[p_{1}\right] \cap \mathrm{N}_{\Omega}\left[p_{2}\right]-\left\{s_{1}, s_{2}, g_{1}, g_{2}\right\}, Y=\mathrm{N}_{\Omega}\left[p_{1}\right]-$ $X-\left\{s_{1}, g_{1}\right\}$, and $Z=\mathrm{N}_{\Omega}\left[p_{2}\right]-X-\left\{s_{2}, g_{2}\right\}$. It is readily verified that $X, Y$, and $Z$ are pairwise disjoint sets, and that we have $X \subset \mathrm{N}_{\Omega}\left[p_{1}\right]$ and $X \subset \mathrm{N}_{\Omega}\left[p_{2}\right]$. Let $M$ be a maximal match from $Y$ to $Z$. Then, we construct $|X|+|M|$ paths from $\left\langle g_{1}, p_{1}\right\rangle$ to $\left\langle g_{2}, p_{2}\right\rangle$ as follows: For every $x \in X$, we have the paths

$$
\left\langle g_{1}, p_{1} \rightarrow x\right\rangle \rightarrow\left\langle x, \operatorname{path}_{\Omega}\left(g_{1}, g_{2}\right)\right\rangle \rightarrow\left\langle g_{2}, x \rightarrow p_{2}\right\rangle,
$$

and for every $(y, z) \in M$, we build the paths

$$
\begin{aligned}
\left\langle g_{1}, p_{1} \rightarrow y\right\rangle & \rightarrow\left\langle y, \operatorname{path}_{\Omega}\left(g_{1}, z\right)\right\rangle \rightarrow\left\langle z, \operatorname{path}_{\Omega}\left(y, g_{2}\right)\right\rangle \\
& \rightarrow\left\langle g_{2}, z \rightarrow p_{2}\right\rangle .
\end{aligned}
$$

The constructions in two typical cases are depicted in Fig. 4 (when $g_{1} \neq g_{2}, p_{1}=p_{2}=p$, and $g_{1}, g_{2} \notin \mathrm{N}_{\Omega}[p]$ ) and Fig. 5 (when $g_{1} \neq g_{2}, \quad p_{1} \neq p_{2}$, and $g_{1}, \quad g_{2} \notin$ $\left.\mathrm{N}_{\Omega}\left[p_{1}\right] \cup \mathrm{N}_{\Omega}\left[p_{2}\right]\right)$.

By definitions of $X$ and $M$, the number of paths given in (4)-(6) above is $1+|X|+|M|$. Considering $\left|\mathrm{N}_{\Omega}\left[p_{1}\right]\right|=d+1, X \subset \mathrm{N}_{\Omega}\left[p_{1}\right], s_{1} \in \mathrm{N}_{\Omega}\left[p_{1}\right]$, and $s_{1} \notin X$, we have $|Y|=d-|X|-1$ if $g_{1} \in \mathrm{N}_{\Omega}\left[p_{1}\right]-X-\left\{s_{1}\right\}$; otherwise, $|Y|=d-|X|$. Similarly, because $\left|\mathrm{N}_{\Omega}\left[p_{2}\right]\right| \geq d+1$, we have $|Z| \geq d-|X|-1$ if $g_{2} \in \mathrm{N}_{\Omega}\left[p_{2}\right]-X-\left\{s_{2}\right\}$; otherwise, $|Z| \geq d-|X|$. Hence, $|M|=d-|X|-1$ or $d-|X|$ due to $|M|=\min \{|Y|,|Z|\}$, and the number $1+$ $|X|+|M|$ of paths is $d$ or $d+1$, as claimed. In particular, if $g_{1} \notin \mathrm{N}_{\Omega}\left[p_{1}\right]$ and $g_{2} \notin \mathrm{N}_{\Omega}\left[p_{2}\right]$, then $|M|=$ $d-|X|$ and the number of paths reaches its maximum of $d+1$.

To complete the proof, we must show the paths defined in (4)-(6) to be pairwise node-disjoint. Because

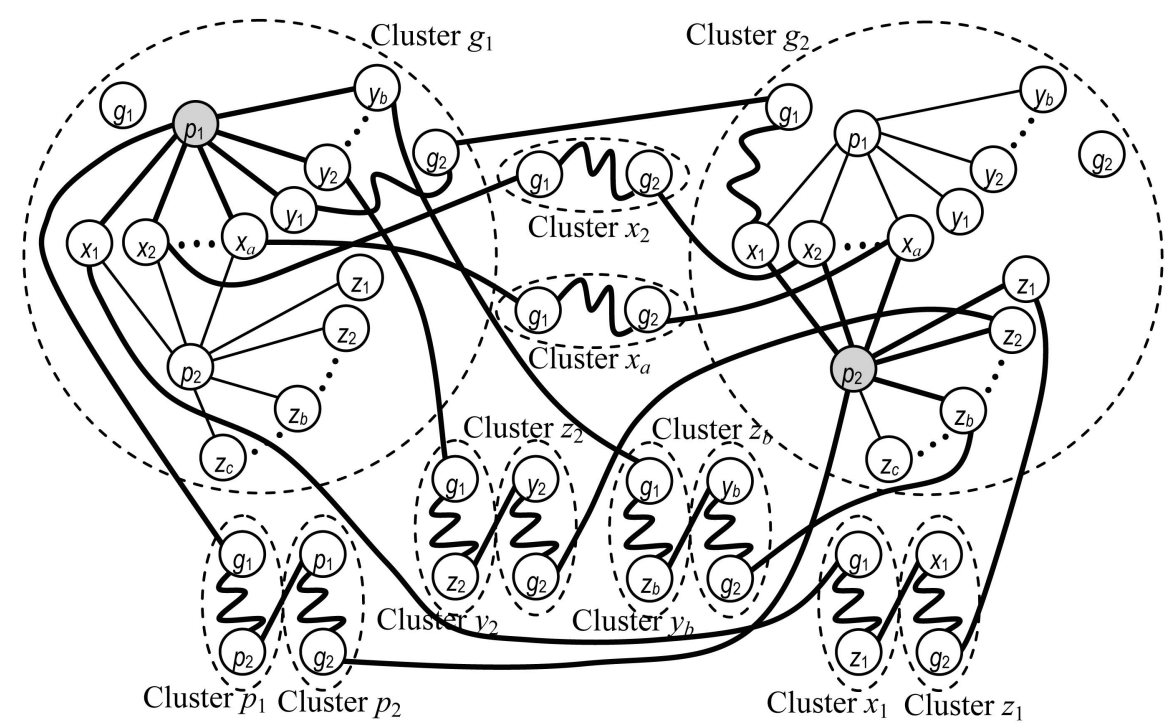

Fig. 5. Constructing $d+1$ node-disjoint paths, shown as heavy lines, in OTIS- $\Omega$ between $\left\langle g_{1}, p_{1}\right\rangle$ and $\left\langle g_{2}, p_{2}\right\rangle$ for $g_{1} \neq g_{2}, p_{1} \neq p_{2}$, and $g_{1}$, $g_{2} \notin \mathrm{N}_{\Omega}\left[p_{1}\right] \cup \mathrm{N}_{\Omega}\left[p_{2}\right]$, where $s_{1}=y_{1}, s_{2}=x_{1}, X=\left\{x_{2}, \ldots, x_{a}\right\}, Y=\left\{p_{1}, x_{1}, y_{2}, \ldots, y_{b}\right\}, Z=\left\{p_{2}, z_{1}, \ldots, z_{b}, \ldots, z_{c}\right\}$, and $M=\left\{\left(p_{1}, p_{2}\right),\left(x_{1}, z_{1}\right)\right.$, $\left.\left(y_{2}, z_{2}\right), \ldots,\left(y_{b}, z_{b}\right)\right\}$. 
$X, Y$, and $Z$ are pairwise disjoint sets, no cluster other than $g_{1}$ and $g_{2}$ is visited by more than one of these paths. So, if any pair of paths were not node-disjoint, they would only intersect in clusters $g_{1}$ or $g_{2}$. However, the latter is impossible, given that all segments of these paths contained in clusters $g_{1}$ and $g_{2}$ are pairwise node-disjoint by construction.

Put together, Lemmas 1 and 2 establish the following result.

Lemma 3. Let $\Omega$ be a connected graph. If $\left\langle g_{1}, p_{1}\right\rangle$ and $\left\langle g_{2}, p_{2}\right\rangle$ are two distinct nodes in OTIS- $\Omega$, then there exist $d$ or $d+1$ node-disjoint $\left(\left\langle g_{1}, p_{1}\right\rangle,\left\langle g_{2}, p_{2}\right\rangle\right)$-paths in OTIS- $\Omega$, where $d=\min \left\{\operatorname{deg}_{\Omega}\left(p_{1}\right), \operatorname{deg}_{\Omega}\left(p_{2}\right)\right\}$.

We are now set to state and prove our main result.

Theorem 3. OTIS- $\Omega$ is maximally fault tolerant if $\Omega$ is a connected graph.

Proof. Let $\Omega$ be a connected graph and $v \in V_{\Omega}$ be a node such that $\operatorname{deg}_{\Omega}(v)=\delta(\Omega)$. By Definition 1, we have $\langle v, v\rangle \in$ $V_{\text {OTIS- } \Omega}$ and $\operatorname{deg}_{\text {OTIS- } \Omega}(\langle v, v\rangle)=\delta(\Omega)=\delta($ OTIS- $\Omega)=d$. Then, from Lemma 3 , there are at least $d$ node-disjoint paths between any two nodes in OTIS- $\Omega$, proving the desired result.

The fault diameter and wide diameter are two important parameters for measuring the reliability and efficiency of interconnection networks. The fault diameter of a $k$-connected graph $G$ is defined as the diameter of the graph that results from $G$ after removing at most $k-1$ nodes. The wide diameter of $G$ is defined as the minimum number $w$ such that there exist $k$ node-disjoint paths of length at most $w$ between any two distinct nodes in $G$. The constructions of Lemmas 1 and 2 provide an upper bound on the fault diameter and wide diameter of OTIS- $\Omega$.

Theorem 4. If $\Omega$ is a connected graph, then both the fault diameter and the wide diameter of OTIS- $\Omega$ are at most $D+4$, where $D$ is the diameter of OTIS- $\Omega$.

Proof. From Lemma 3, it suffices to show that the length of every constructed path is at most $D+4$. Let $D(\Omega)$ be the diameter of $\Omega$. Then, we have $D=2 D(\Omega)+1$, where $D$ is the diameter of OTIS- $\Omega$. Since the length of path $_{\Omega}(u, v)$ is at most $D(\Omega)$ for all $u, v \in V_{\Omega}$, it is readily verified that the length of every path in (1)-(3) in the proof of Lemma 1 and (4)-(6) in the proof of Lemma 2 is at most $2 D(\Omega)+5$, i.e., $D+4$, as claimed.

\section{Conclusions}

We have shown that any OTIS (swapped) network built of a connected basis network enjoys the maximal fault tolerance property. This result supersedes a number of maximal fault tolerance proofs for networks that are special cases of OTIS networks and will obviate the need for additional proofs for other basis networks of potential interest. One example is the recent result of Day [3] on optical transpose $k$-ary $n$-cube networks, which can be stated as a corollary to our Theorem 3. Our result is stronger than the corresponding result of [2] (restated in our Theorem 2). For example, in an OTIS network with its basis graph $\Omega$ depicted in Fig. 6,

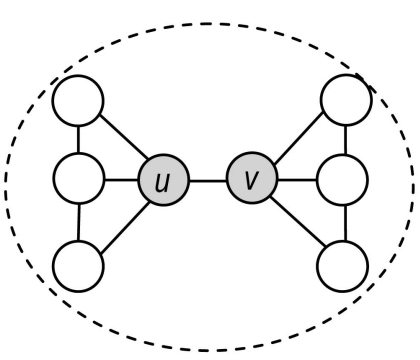

Fig. 6. An example basis graph $\Omega$ with $\delta(\Omega)=2$.

Theorem 2 suggests no guaranteed node-disjoint paths between nodes $\left\langle g_{1}, u\right\rangle$ and $\left\langle g_{2}, v\right\rangle$ in OTIS- $\Omega$ for all $g_{1}$ and $g_{2}$, while by our Lemma 3 , there are four or five such paths, depending on the cluster numbers $g_{1}$ and $g_{2}$ and the numbering of the neighbors of $u$ and $v$ in Fig. 6 .

Intuitively, the reason for both maximal fault tolerance and the corresponding construction of node-disjoint paths between nodes in OTIS- $\Omega$ being independent of the basis network $\Omega$, and only requiring its connectivity, is due to the intercluster connection scheme. If we consider each cluster as a supernode, then all supernodes are connected as a complete graph via intercluster links. It is this intercluster connectivity that enriches the node connectivity of an OTIS network compared with its basis graph. Therefore, our result should be viewed as a new attribute arising from the OTIS architecture and not from its basis graph. This result further confirms the suitability of OTIS architectures for multiprocessor interconnection networks, benefiting from both optical and electronic technologies.

One possible avenue for future research is determining whether fault tolerance can be improved by rendering a swapped (OTIS) network regular. As defined, for a regular $n$-node degree- $d$ basis network, $n^{2}-n$ nodes of the associated swapped network have degree $d+1$, with the remaining $n$ nodes having degree $d$. When $n$ is even, the $n$ nodes that do not possess intercluster links can be connected pairwise to make all nodes of degree $d+1$. Can this be done in a way to guarantee the existence of $d+1$ node-disjoint paths for every node pair?

\section{ACKNOWLEDGMENTS}

The research of the first two authors was supported by the Natural Science Foundation of Guangdong Province, P.R. China.

\section{REFERENCES}

[1] A. Al-Ayyoub, A. Awwad, K. Day, and M. Ould-Khaoua, "Generalized Methods for Algorithm Development on Optical Systems," I. Supercomputing, vol. 38, pp. 111-125, 2006.

[2] K. Day and A. Al-Ayyoub, "Topological Properties of OTISNetworks," IEEE Trans. Parallel and Distributed Systems, vol. 14, no. 4, pp. 359-366, Apr. 2002.

[3] K. Day, "Optical Transpose $k$-ary $n$-cube Networks," J. Systems Architecture, vol. 50, pp. 697-705, 2004.

[4] P.K. Jana and B.P. Sinha, "An Improved Parallel Prefix Algorithm on OTIS-Mesh," Parallel Processing Letters, vol. 16, no. 4, pp. 429-440, 2006.

[5] A. Krishnamoorthy, P. Marchand, F. Kiamilev, and S. Esener, "Grain-Size Considerations for Optoelectronic Multistage Interconnection Networks," Applied Optics, vol. 31, no. 26, pp. 5480-5507, 1992. 
[6] G. Marsden, P. Marchand, P. Harvey, and S. Esener, "Optical Transpose Interconnection System Architecture," Optical Letters, vol. 18, pp. 1083-1085, 1993.

[7] P.K. Jana, "Polynomial Interpolation and Polynomial Root Finding on OTIS-Mesh," Parallel Computing, vol. 32, pp. 301-312, 2006.

[8] A. Osterloh, "Sorting on the OTIS-Mesh," Proc. 14th Int'l Parallel and Distributed Processing Symp. (IPDPS '00), pp. 269-274, 2000.

[9] B. Parhami, "The Hamiltonicity of Swapped (OTIS) Networks Built of Hamiltonian Component Networks," Information Processing Letters, vol. 95, pp. 441-445, 2005.

[10] B. Parhami, "Swapped Interconnection Networks: Topological, Performance, and Robustness Attributes," J. Parallel and Distributed Computing, vol. 65, pp. 1443-1452, 2005.

[11] S. Rajasekaran and S. Sahni, "Randomized Routing, Selection, and Sorting on the OTIS-Mesh," IEEE Trans. Parallel and Distributed Systems, vol. 9, no. 9, pp. 833-840, Sept. 1998.

[12] C.-F. Wang and S. Sahni, "Basic Operations on the OTIS-Mesh Optoelectronic Computer," IEEE Trans. Parallel and Distributed Systems, vol. 9, no. 12, pp. 1226-1236, Dec. 1998.

[13] C.-F. Wang and S. Sahni, "Image Processing on the OTIS-Mesh Optoelectronic Computer," IEEE Trans. Parallel and Distributed Systems, vol. 11, no. 2, pp. 97-109, Feb. 2000.

[14] C.-F. Wang and S. Sahni, "Matrix Multiplication on the OTISMesh Optoelectronic Computer," IEEE Trans. Computers, vol. 50, no. 7, pp. 635-646, July 2001.

[15] C.-F. Wang and S. Sahni, OTIS Optoelectronic Computers, Chapter 5 in Parallel Computation Using Optical Interconnection, K. Li, Y. Pan, and S.Q. Zhang, eds., pp. 99-116, Kluwer Academic Publishers, 1998.

[16] D.B. West, Introduction to Graph Theory, pp. 149-169. Prentice-Hall, 2001.

[17] C.-H. Yeh and B. Parhami, "Swapped Networks: Unifying the Architectures and Algorithms of a Wide Class of Hierarchical Parallel Processors," Proc. Int'l Conf. Parallel and Distributed Systems (ICPDS '96), pp. 230-237, 1996.

[18] F. Zane, P. Marchand, R. Paturi, and S. Esener, "Scalable Network Architectures Using the Optical Transpose Interconnection System (OTIS)," J. Parallel and Distributed Computing, vol. 60, no. 5, pp. 521-538, 2000.

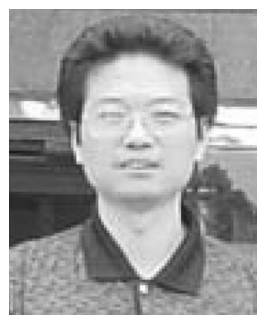

Weidong Chen received the BS degree in mathematics from Huazhong Normal University, Wuhan, P.R. China, in 1991 and the MS degree in computer science from Huazhong University of Science and Technology, Wuhan, in 1997. He is an associate professor in the Department of Computer Science, South China Normal University, Guangzhou, P.R. China. He is currently a PhD candidate in computer science at South China University of Technology, Guangzhou. His research interests include graph theory, optimization theory, approximation algorithms, and parallel computing.

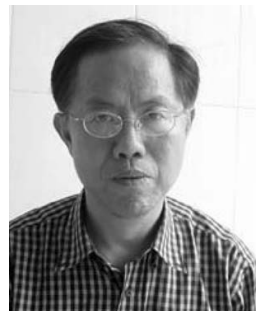

Wenjun Xiao received the $\mathrm{PhD}$ degree in mathematics from Sichuan University, Chengdu, P.R. China, in 1989. He is currently a professor in the Department of Computer Science, South China University of Technology, Guangzhou, P.R. China. His research interests include discrete mathematics, parallel and distributed computing, complex networks, and software architecture. He has published more than $60 \mathrm{pa}-$ pers in international conferences and journals, including the IEEE Transactions on Computers and the IEEE Transactions on Parallel and Distributed Systems on these topics since 1985.

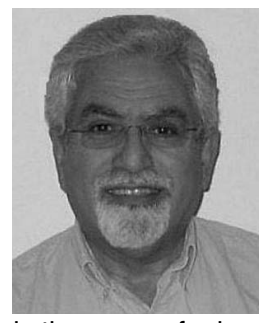

Behrooz Parhami received the $\mathrm{PhD}$ degree in computer science from the University of California, Los Angeles, in 1973. He is currently a professor in the Department of Electrical and Computer Engineering, University of California at Santa Barbara. His research interests include parallel architectures and algorithms, computer arithmetic, and reliable computing. In his previous position at Sharif University of Technology, Tehran, Iran (1974-1988), he was also involved in the areas of educational planning, curriculum development, standardization efforts, technology transfer, and various editorial responsibilities, including a five-year term as editor of Computer Report, a Persianlanguage computing periodical. His technical publications include more than 250 papers in journals and international conferences, a Persianlanguage textbook, and an English/Persian glossary of computing terms. Among his latest publications are two graduate-level textbooks on parallel processing (Plenum, 1999) and computer arithmetic (Oxford, 2000), and an introductory textbook on computer architecture (Oxford, 2005). He is a fellow of the IEEE and the IEEE Computer Society, a chartered fellow of the British Computer Society, a member of the ACM, and a distinguished member of the Informatics Society of Iran, for which he served as a founding member and president during 1979-1984. He also served as chairman of the IEEE Iran Section (1977-1986) and received the IEEE Centennial Medal in 1984.

$\triangleright$ For more information on this or any other computing topic, please visit our Digital Library at www.computer.org/publications/dli. 